\title{
Bernard Juillerat. Lettres à un jeune ethnologue
}

\section{Philippe Peltier}

\section{(2) OpenEdition}

\section{Journals}

Édition électronique

URL : http://journals.openedition.org/jso/6001

DOI : 10.4000/jso.6001

ISSN : 1760-7256

\section{Éditeur}

Société des océanistes

\section{Édition imprimée}

Date de publication : 15 décembre 2010

Pagination : 41-46

ISBN : 978-2-85430-027-7

ISSN : 0300-953x

Référence électronique

Philippe Peltier, «Bernard Juillerat. Lettres à un jeune ethnologue », Journal de la Société des Océanistes [En ligne], 130-131 | 2010, mis en ligne le 15 décembre 2010, consulté le 11 juin 2020. URL : http:// journals.openedition.org/jso/6001; DOI : https://doi.org/10.4000/jso.6001

(C) Tous droits réservés 


\section{Bernard Juillerat Lettres à un jeune ethnologue ${ }^{1}$}

En 1991, quelques semaines avant de partir dans la basse vallée du Sépik en Papouasie Nouvelle-Guinée, pour un second séjour, je remis à Bernard Juillerat les "notes de terrain" de ma première enquête ethnographique chez les Adjirab, un groupe voisin des Banaro. Quelques mois plus tard, Bernard m'envoya plusieurs lettres de commentaires soigneusement tapées à la machine.

Bernard aurait probablement considéré que la place de ces lettres, simple échange entre deux chercheurs, l'un aguerri et ayant déjà publié des études ethnographiques importantes, l'autre encore balbutiant vis-à-vis de la méthode et des objectifs de sa recherche, était de rester sagement rangées au fond d'un tiroir. Il cultivait la discrétion et était peu enclin aux anecdotes.

Cependant, la publication d'extraits de cette correspondance est née d'un désir de donner à entendre à nouveau sa voix. Une voix amicale mais ferme qui, par-delà ses conseils, ses suggestions et ses questions, parle de méthode d'enquête et d'objectifs de recherche. Ces lettres témoignent combien Bernard s'est toujours gardé d'appliquer une quelconque grille de lecture dans ses travaux. Pour lui, toute analyse ethnographique devait s'adosser à une longue, précise et patiente collecte de données. La théorie devait découler des faits. Il le répétait souvent : "Ce sont les Yafar qui m'ont mené à Freud».

Ces lettres relèvent, comme il le reconnaît luimême, de la technique de l'électrochoc, technique qu'il appliquait souvent, il faut l'avouer, avec virtuosité. Quelques années plus tôt, j’en avais expérimenté toute l'efficacité.

Un jour, alors que nous prenions un café avant de nous rendre au séminaire de recherche sur l'Océanie dont il était responsable, il m'avait dit tout de go: "Tu as trop la trouille, tu ne partiras jamais sur le terrain! Q Quelques semaines plus tard, j'étais dans l'avion.

La première lettre fut une douche froide. Tout chercheur est particulièrement vulnérable sur le terrain. Les lettres suivantes, réponses à mes explications ou mes justifications probablement véhémentes, sont attentives et soucieuses de ne pas blesser. Bernard avait pleinement conscience que la recherche est un exercice difficile et sans fin.

Confessons cependant un fait. Il est un point sur lequel je n'ai pas suivi son conseil : je n'ai jamais eu de radio sur le terrain. Il démontre que Bernard, par-delà sa passion pour son métier, était curieux du monde et attentif aux autres.

Philippe Peltier*, Paris, avril 2010

$*$

\section{Paris, 24/8/91}

\section{Cher Philippe,}

J'ai bien reçu ta lettre et ton envoi d'Australie (Gewertz) ainsi que trois envois de PNG. Un immense merci pour la peine que tu t'es donnée pour rechercher, trouver, photocopier tout cela. Note ce que tu as dépensé pour que je te le rembourse à ton retour. Les mythes s'accumulent (tu aurais pu les envoyer par bateau comme je te l'avais dit...) mais je ne pourrai m'y plonger tout de suite. Un texte sur la guerre en West Papua pour Ethnies ${ }^{2}$, puis l'article sur Thurnwald ${ }^{3}$ pour Gradhiva [Juillerat, 1993: 15-40], j'ai rapporté de Berlin quelques photos pas trop mauvaises par Th[urnwald] sur la Keram. Le texte de Bengo clarifie la généalogie Eichorn [Bengo, 1974 : 36-38] ; celui de Laycock

1. Je remercie Michèle Juillerat pour son autorisation à publier ces quelques lettres. Entre crochets et en notes de bas de page, j'ai rajouté quelques compléments nécessaires à la compréhension.

2. Dans une lettre datée du 9 juillet, Bernard précisait que, suite à une mésentente, cette publication était reportée fin 1993. Il semble que cet article n'ait jamais été publié. À cette époque, il travaille à un autre article qui se transformera petit à petit en un livre sur la cérémonie yangis ( $c f$. L'avènement du père, publié en 1995 au presses du CNRS), précisant non sans humour retenu «On n'en finit pas de réfléchir sur le symbolisme quand on a des matériaux suffisamment sûrs... Saturé de symbolique "œdipienne" et d'autres, j'ai hâte de retourner rendre visite à Thurnwald que j'ai dû laisser de côté depuis plusieurs mois ».

3. Richard Thurnwald, ethnologue né à Vienne en 1869 et mort en 1954. Il séjourna dans le Sépik entre 1912 et 1915 . Il publia deux études sur les Banaro, en 1916 et 1921. La lecture de ces études a décidé Bernard à travailler dans ce groupe.

* Anthropologue, conservateur en chef du patrimoine responsable de l'unité patrimoniale Océanie Insulinde, musée du quai Branly, philippe.peltier@quaibranly.fr 
[1975] n'apporte en fait rien de nouveau par rapport à sa publication principale sur le Sépik (j'avais cru comprendre que le texte traitait de la région Sépik-Ramu).

Je viens de lire tes «notes de terrain 87 ». La carte linguistique de la région a l'air plus complexe que celle que donne Laycock; confusion sur le terme Aiom (qu'il orthographie aion) et distingue de la langue adjora, les Banaro distinguent pour le Porapora deux langues : awa et awo!

- L'organisation spatiale dans les m[aisons] des h[ommes] semble différente que chez les Banaro, par le fait notamment que tu as quatre semi-moitiés.

- Sous ton entrée "Nduara », cette histoire de garamut $^{4}$ pourrait se rattacher au garamut conservé par les Bobten (vois-le si tu y passes) ${ }^{5}$ et relié au mythe banaro que je t'ai donné et sur le mythe kambot (pardon : tin dama !) que Lupu a publié dans le JSO [Lupu, 1973 : 313-323].

- Sur les trous d'eau qui cachaient les sculptures et où l'on sacrifiait les enfants, cela laisse évidemment rêveur. Le sacrifice d'enfant (de quel âge ?) s'apparenterait-il à la fois à une forme d'infanticide? Je suppose que tu vas piocher sur le thème pour en savoir plus! Quant aux objets de bois conservés dans l'eau, cela me rappelle la convocation et le renvoi à un étang des tamba$r{ }^{6}{ }^{6}$ de Toko ${ }^{7}$. Je vois aussi dans ton chapitre " Colonisation », que les gens jetaient dans des trous d'eau les objets qu'ils ne voulaient pas voir emporter par le missionnaire ou le marchand (y aurait-il un rapport entre les deux choses : une manière de garder « chez soi » les tambaran et leurs représentations. Détail technique: le bois devait pourrir rapidement dans l'eau, non?).

- Ton bref commentaire sur les « charismatiques » paraît intrigant : j'ai l'impression qu'il doit y avoir des mouvements semblables dans la région. On m'a signalé un culte dans le Grass Country (rive gauche de la Keram) étudié par le fils ou le frère d'un missionnaire protestant pour un MA à Melbourne. Je lui ai écrit mais n'ai reçu aucune réponse... Tu devrais mettre le paquet sur ce sujet.

J'avais bien sûr aussi reçu tes commentaires sur mon manuscrit, mais je crois qu'on en avait un peu parlé au téléphone avant ton départ.
Concernant la clarté de mon résumé sur l'analyse de Thurnwald (chapitre 1), je crois que cela est clair, bien qu'évidemment un peu compliqué. Je comprends bien que tu n'as pas disposé de temps nécessaire pour digérer le tout!

Maintenant que te revoilà en prise directe avec la réalité ethnographique, tu vas pouvoir renouer des liens plus étroits avec tes informateurs et reprendre ton Journal ethnographique. Personnellement, je pense qu'il vaut mieux séparer le Journal (très utile à différents titres) et les notes scientifiques; sinon on risque de donner à ses matériaux une allure anecdotique due à l'humeur de l'interlocuteur (et à la sienne propre) ce jour-là ou à des mini-événements aléatoires divers. Cela permet aussi de mieux conduire les entretiens, c'est-à-dire d'en faire de véritables séances de travail, et non pas seulement des conversations (qui doivent rester parallèlement et ont bien entendu leur charme et leur nécessité psychologique). Passé un certain niveau dans la connaissance d'une société, je crois qu'il est bon que nous fassions comprendre à nos interlocuteurs locaux que notre boulot est quelque chose d'important (au moins pour nous), de sérieux et de difficile, quelque chose qui exige un minimum de concentration et de réflexion. Il est d'ailleurs possible à ce niveau (quand on se connaît déjà bien) de faire assimiler aux meilleurs informateurs les principes fondamentaux des problématiques anthropologiques, les questions qu'on se pose (sur l'autorité, les échanges, les règles de mariage, la structure dualiste, les cultes anciens, etc.) ; cela permet que les interlocuteurs privilégiés s'intéressent davantage au travail et fournissent des informations plus exactes et mieux ciblées. Cela suppose évidemment de s'imposer aussi à soi-même quelques principes méthodologiques et de s'y tenir; par exemple, ne pas laisser passer un commentaire pas clair mais au contraire de le traiter «à chaud» en ne s'autorisant à passer outre que lorsque la question a été explicitée au mieux, de se ménager des moments (le soir pas exemple) de réflexion sur les matériaux déjà en main et de préparer pour le lendemain des entretiens très « pointus » à base sinon de questionnaires du moins de questions préparées à l'avance et fondées sur les dernières séances de travail. Cela donne une continuité aux séances et permet d'éviter l'impressionnisme, voire le pointillisme-

4. Garamut : tambours à fente (pidgin de Nouvelle-Guinée).

5. Le tambour existait bien encore mais les poignées en avaient été découpées pour être vendues à un antiquaire de passage. Le corps du tambour ne portait pas de motifs, rendant toute étude sur son origine impossible.

6. Tambaran : les esprits-ancêtres (pidgin de Papouasie Nouvelle-Guinée).

7. Village de la région Banaro où Bernard Juillerat a mené son enquête. Voir à ce sujet La révocation des Tambaran. À cette date, ce livre n'était pas encore publié. Bernard m'avait donné le tapuscrit à lire quelques semaines avant mon départ. 
ou mieux le surréalisme ! - ethnographique que rappellent parfois les matériaux bruts (quand on relie nos notes par hasard). Le fait d'obtenir des séances de travail avec le ou les mêmes hommes de façon régulière (ou tous les jours pendant une ou deux semaines) renforce cette continuité. (C'est pourquoi j'ai toujours rémunéré de telles séances de travail, avec en rab un bon repas à base de tin mit $^{8}$ à midi !). L'enregistrement sur bande est certainement bien pratique et je l'ai beaucoup utilisé ; il y a cependant un petit danger dont il suffit d'être conscient pour l'éviter : la paresse d'intervention à chaud précisément, en se disant que l'on verra plus tard en réécoutant la bande. Ici, un clignotant doit s'allumer sinon sur le magnéto du moins dans la tête de son manipulateur. Ce qui est le plus important, c'est ce que l'on capte dans son cerveau sur le moment, non pas ce qui est enregistré. Cela permet de comprendre que l'information n'est pas dans une phrase ou un topo de telle ou telle personne, mais bien dans le sens qui se constitue entre l'interviewé et l'intervieweur au cours de l'échange, ou plutôt des échanges successifs, et de façon cumulative. C'est à force de remettre le même sujet " sur le tapis » des entretiens formels (quitte à ce que l'informateur en soit irrité !) qu'on finit par le traiter de façon satisfaisante.

Excuse ces propos quelque peu didactiques (je veux te faire profiter de ma "grande expérience »!!) ; après tout, chacun ses méthodes, mais cela peut peut-être servir...

Je termine ce feuillet en t'imaginant à l'écoute de ta radio pour tenter de suivre les événements de Moscou (et ce n'est peut-être pas terminé). Lorsque j'étais chez les Banaro, j'ai suivi de cette façon les événements de Roumanie et la chute du mur ; en 86 je suivais les attentats à Paris, en 73 les bombardements américains au Cambodge et en 68, au Cameroun, les événements de mai ! La radio est un autre instrument indispensable du terrain.

Si tu as besoin de quoi que ce soit, n'hésite pas bien sûr (livres, photocopies, médicaments...). Je t'envoie cette lettre à l'ancienne adresse (que tu as mise sur l'enveloppe)...

Amitiés

Bernard

PS manuscrit: Amitiés de Michèle. Nous avons fait une virée en Tchécoslovaquie/Hongrie très agréable.

\section{Cher Philippe,}

Je te croyais déjà noyé dans la Bien ${ }^{9}$, n'ayant pas de nouvelles. Et je lis que tu as pu croire que ma lettre avait quelque intention malfaisante! Comment est-ce possible de penser qu'elle ait été autre chose qu'une communication franche et amicale ??? Je sais que je peux être un peu direct parfois, malgré ma difficulté générale à communiquer, mais aurais-tu oublié que je suis Vaudois ? Si je me permets de te faire part de quelques commentaires qui me sont venus à la lecture de tes notes, c'est simplement qu'il m'a semblé que tu te trouvais dans la situation où j'étais moi-même en 1971, c'est-à-dire avec un tas d'informations partielles et fragmentées n'aboutissant pas à une problématique précise. Je connais cela puisque je suis passé par là lors de mon premier séjour chez les Yafar. Donc, rassure-toi, je ne faisais que réfléchir à tes inévitables difficultés d'enquête et à ta solitude d'ethnographe de terrain. Des notes, ce sont des notes et rien de plus, ce n'est pas n'est pas une œuvre littéraire ni un travail scientifique achevé ! C'est quelque chose de provisoire qui demande par définition à être repris et transformé. Il est vrai qu'en France (ou peut-être seulement chez les Parisiens ?), on n'ose pas se parler franchement de son travail respectif : ça ne se fait pas. On se fait des compliments ou bien l'on se tait. On a beau se connaître (je parle en général) depuis quinze ou vingt ans, eh bien non, on continue à rester dans le vague de peur de vexer l'autre. J'ai déjà remarqué cela souvent. Mais je trouve que c'est dommage et que sous cette "diplomatie » se cache finalement un manque de confiance et d'amitié. De mon côté, j'attends toujours de nos chers collègues qu'à l'occasion ils me disent « amicalement » ce qu'ils ont pensé des Enfants $d u$ Sang ou d'Edipe chasseur. Mais je n'entends que le silence d'une politesse feutrée et typiquement parisienne.

Cela dit, tu ne donnes pas beaucoup de détails sur ton mode de vie. Pirogue personnelle? Moteur? Maison des hommes ou maison de l'ethnographe tout seul? Cafard ou enthousiasme? Je vois aussi que ton adresse est à Wewak, ce que me laisse supposer que la poste d'Angoram ${ }^{10}$ est toujours fermée et qu'à

8. Conserve de viande (pidgin de Papouasie Nouvelle-Guinée). Cette nourriture est particulièrement valorisée dans les villages. Il n'est pas rare d'en voir apparaître dans les échanges cérémoniels.

9. Bien : rivière du bas Sépik que l'on doit emprunter pour se rendre dans les villages Adjirab.

10. Créé par les Allemands en 1913 comme poste de gendarmerie, Angoram est un centre administratif desservant la basse vallée du Sépik. En 1991, la poste et le supermarché étaient effectivement fermés suite à des actes de banditisme. 
Marienberg ${ }^{11}$ il n'y a pas grand monde... Qu'en est-il du problème «raskol ${ }^{12}$ »? Le Monde a inséré dix lignes il y a un mois pour annoncer que le parlement de PNG avait rétabli la peine de mort... Par ailleurs, je travaille à un article sur la « guerre » en Irian Jaya, que m'a demandé Ethnies une fois de plus. On n'a pas beaucoup d'informations récentes. Un ancien chef de l'OPM ${ }^{13}$, Salosa, qui avait été extradé par la PNG où il était depuis 79 , a été condamné à perpète en 90 et a été retrouvé mort dans la forêt après s'être « évadé » (selon les versions officielles), à proximité de la prison de Waena près de Jayapura. Les gens ayant participé au lever du drapeau indépendantiste en 88 ont été condamnés dès 89 à des peines allant jusqu'à 20 ans pour l'organisateur principal, le $\mathrm{D}^{\mathrm{r}}$ Wingti, intellectuel pacifiste (sa femme a eu 13 ans pour avoir cousu le drapeau) ; plus de trente personnes ont été condamnées au total pour cette même montée des couleurs. Selon Amnesty, il y a actuellement cent trente Mélanésiens dans les prisons indonésiennes (dont près de la moitié à Java, loin de leurs familles), dont 80 sont des prisonniers d'opinion. Si tu tombes sur des articles intéressants dans le Post Courier ou le $P N G$ Times, concernant ce problème, rapporte-les s'il te plaît.

Iras-tu chez ces chers Banaro ? Demande-leur ce que j'ai totalement oublié de leur demander, à savoir si leurs ancêtres chassaient les têtes ${ }^{14}$. J'ai l'impression que cela ne concernait que les Banaro de l'aval : il me semble que les Toko m'en auraient dit quelque chose à travers toutes leurs descriptions de haus tambaran ${ }^{15}$ et de guerres. Il faut croire que le problème ne me préoccupait guère.

Deborah Gewertz a publié un troisième livre sur les Chambri. Intitulé Twisted histoires, altered contexts ${ }^{16}$. Elle y traite du changement culturel, du tourisme et de la façon dont un big man s'en sert pour accroître son prestige, des Chambri de Wewak, etc. Si tu veux, je te l'envoie, dis-le moi (j'ai fait un c.r. pour l'Homme ${ }^{17}$ ).

Em tasol ${ }^{18}$. À propos, as-tu trouvé de nouvelles versions de mythes ? En lisant la littérature à ce sujet, je me suis aperçu que le thème des deux frères dont le cadet à une relation avec la femme de l'ainé (l'histoire du dessin) et échappe ensuite à la vengeance de l'aîné (l'histoire du pieu planté dans le trou) a une très large diffusion, jusqu'au Vanuatu!

Amitiés

Bernard.

$*$

Paris, 22/1/92

\section{Cher Philippe}

Tu auras reçu le Tuzin et l'article de Joséphides ${ }^{19}$.

Ne t'inquiète pas : tu ne m'as pas « blessé » du tout. J'ai seulement pensé après ta première lettre que je n'avais pas été assez fin et que mon message n'était pas passé. Mais ta seconde lettre m'a pleinement rassuré ! Maintenant je peux dévoiler mon jeu! J'avais cru sentir en lisant tes notes mais aussi en parlant avec toi ici avant ton départ un léger flottement dans tes problématiques anthropologiques. Et me souvenant des miennes au début des années 70 - je me suis dit qu'il fallait un petit électro-choc pour te remettre dans une voie plus claire, mieux balisée. Voilà. Enfin si cela t'a servi à quelque chose, tant mieux. J'espère que tu te rendras compte à quel point l'enquête ethnographique peut être difficile, non-seulement techniquement mais psychologiquement. Chez les Banaro, j'avais plutôt le cafard, je peux te le dire !

[...]

Dis-moi un peu l'avance quand tu penses rentrer. J'aurai encore un ou deux titres à l'IPNGS ${ }^{20}$ (mythes), à te demander.

Bon temps

Amitiés

Bernard.

$*$

11. Marienberg : centre installé par la mission catholique allemande sur la rive gauche du bas Sépik. En 1991, il n’y avait plus de prêtre mais une sœur, Sister Marianna, qui dirigeait un centre de soins.

12. Raskol: bandit, criminel (pidgin de Papouasie Nouvelle-Guinée. Le terme apparaît dans les années 1970).

13. opm : Organisasi Papua Merdeka (Organisation de libération de la Papouasie).

14. Lors de mon séjour, je ne suis pas retourné chez les Banaro. La question est restée sans réponse.

15. Haus tambaran: maison des esprits (pidgin de Papouasie Nouvelle-Guinée). Le terme s'applique plus particulièrement aux maisons des hommes lorsque des cérémonies sont en cours.

16. Livre publié avec Frederick K. Errington.

17. Ce compte rendu est paru dans L'Homme 34, 131, pp. 191-193.

18. Expression de pidgin mélanésien qui peut se traduire par « c'est fini » ou « c'est assez ».

19. Je n'ai hélas jamais reçu cet envoi...

20. Institut of Papua New Guinea Studies. Fondé en 1974, l'Institut a pour mission de recueillir et d'étudier les traditions orales et la musique. 
15/5/92 J'ai été intrigué par ta relation se-emba (père-

\section{Cher Philippe,}

Bien reçu ta lettre du 23 avril. Lemonnier a reçu la sienne juste après. [...]

C'est comment d'avoir des rhumatismes? La saison des pluies et le fait de vivre les pieds (ou davantage que les pieds) dans l'eau est sans doute pour quelque chose. Enfin j'espère que ta bonne-sœur de Marienberg t'aura guéri et que tu es de nouveau à pied d'œuvre. Ne t'inquiète pas pour les Banaro et n'y va surtout pas pour moi : mon manuscrit a été lu par les nouvelles commissions du CNRS pour les publications (responsable pour l'ethno: I. Chiva) qui fait lire les manuscrits à l'extérieur (de la commission) : c'est à Lemonnier qu'est revenu cette tâche. Il a fait un bon rapport bien sûr et j'attends des nouvelles du CNRS maintenant (des sous, un éditeur?).

Tes versions " secrètes" des mythes ne sont peut-être qu'un premier stade d'ouverture vers les versions ésotériques plus intéressantes (s'il y en a). Les versions secrètes yafar de mythes par ailleurs publics (c'est-à-dire existant en version profane, ce qui n'est pas le cas de tous les mythes secrets) n'étaient pas toujours très intéressantes au premier abord, mais les symboles relevés entraient dans un système de significations et cosmologique plus vaste (par exemple le cocotier totémique mère), ouvraient des perspectives entre mythes ou entre mythe et rituel, etc. Je crois qu'un mythe doit être d'abord interprété en soi (l'histoire de ses personnages, le « héros » comme sujet), puis dans le contexte culturel plus large. Essaie de faire parler tes spécialistes sur certains symboles ou personnages; même poser des questions naïves du genre "Pourquoi cela, pourquoi pas plutôt ceci ?, etc. ». Et surtout des questions sur les rapports de parenté qui apparaissent dans les récits, entre frères, et entre parents et enfants (filiation). Si les gens ont du mal à parler, il faudrait savoir ce qu'ils craignent : que d'autres (individus, clans etc.) connaissent le mythe qu'ils ne doivent pas connaître, des sanctions surnaturelles, révéler quelque chose à un étranger? Tu peux les assurer de ta discrétion par rapport aux Adjora.

Quant aux influences occidentales (notions, âmes, etc.) et notamment missionnaires, je sais qu'il n'est pas toujours facile de les séparer de ce qui est «traditionnel», d'autant que les gens eux-mêmes ne le savent pas toujours. Les Toko revendiquaient que certains éléments de l'histoire biblique existaient déjà chez eux avant le contact, et que la Bible n'a fait que confirmer qu'ils avaient « raison ». fils), voilà qui sonne nouveau à mes oreilles. Cela est intrigant surtout dans une société matrilinéaire! N'oublie pas de voir comment se transmet la relation aux générations suivantes, comment elle se distribue lorsqu'il y a plusieurs fils (s'il s'agit bien d'une relation véritable entre père et fils ??) et quelle est la position de la mère dans tout cela (y a-t-il quelque chose de correspondant pour mère/fille, conformément à l'idée de «sex affiliation" (Williams) qui caractérise le système de descendance Banaro ?). S'agit-il seulement de forme d'échanges ou y a-t-il une dimension cosmologique ? Tu te souviens peutêtre dans les Enfants du sang, de l'axe est (fils) louest (père) et des premières maisons construites dans un nouveau hameau, la "maison du fils » à l'est et la «maison du père » à l'ouest. Mais il ne s'agit là pas de vrais pères et de fils, mais de clans et lignées incarnant ces positions parentales symboliques. Le tout s'inscrivant sur le sol (terre-mère), soit sur le ventre maternel (la place du village). Une façon d'inscrire la filiation masculine hors du corps féminin ou pourtant le père se transforme en fils. Peut-être trouveras-tu ces lieux de la culture où le système social (descendance, etc.) rejoint la cosmologie.

Je n'ai pas bien compris ce que tu appelles " procès ». Je suppose une discussion purement locale à la recherche d'un consensus ou d'une condamnation morale interne au groupe ? Historique de la pratique (postcoloniale ou plus ancienne?).

Je vois que la corruption n'existe pas qu'en France (ou en Suisse!). Bien sûr que je savais pour la PNG. Ce pauvre tiers-monde! J'ignorais en outre que les archives de l'East Sepik Province avaient brûlé. S'agit-il de cette petite maison avec un escalier extérieur pour atteindre le premier étage ? (c'est là que j'avais consulté les archives démographiques sur les Banaro).

$[\ldots]$

Andrew Strathern est à Paris pour quelques semaines, invité par Godelier.

Bon courage pour la fin du terrain, profites-en! Je veux dire sur le plan travail. Envoie encore un petit mot pour dire si tu es de nouveau sur pied.

Sincères amitiés

Bernard.

PS. Je suis en pleine lecture des épreuves de Shooting the Sun, le bouquin américain sur Yangis.

PS2. Je ne me suis pas occupé de mon travail comparatif sur les mythologies depuis plusieurs mois, aussi je ne sais plus où j'en suis pour ma documentation. Je n'ai donc pas de nouveaux achats à te demander. Simplement si tu vois 
quelque chose de nouveau et de qualité, surtout à l'IPNGS par exemple, prends-le. Je te rembourserai tout cela.

\section{RÉFÉRENCES CITÉES}

Bengo P., 1974. Georg Eichorn amongst the Korogopa of the Keram River, Oral History (Port Moresby) 2, 5, pp. 36-38.

Gewertz Deborah et Frederick K. ERrington, 1991. Twisted histoires, altered contexts, Cambridge, Cambrige University Press.
JuiLlerat Bernard, 1993. Thurnwald et la Mélanésie, Gradhiva 14, pp. 15-40.

-, 1993. La révocation des Tambaran. Les Banaro et Richard Thurnwald revisités, Paris, CNRs Éditions.

—, 1995. L'avènement du père, Paris, presses du CNRS.

Laycock D. et J. Z'GragGen, 1975. The Sepik-Ramu Phylum, Pacific Linguistics, séries C, 38.

LuPU François, 1973. Sur la circulation de trois objets dans la basse vallée du Sepik, Journal de la Société des Océanistes 29, 40, pp. 313-323. 